\title{
Dissection-enabled scaffold-assisted resection (DeSCAR): a novel technique for resection of residual or non-lifting gastrointestinal neoplasia of the colon, expanded experience and follow-up
}

\section{다)(i) $(5)$}

\author{
Authors \\ Institution \\ University of Chicago Medical Center, Center for \\ Endoscopic Research and Therapeutics (CERT), Chicago, \\ Illinois, United States \\ submitted 18.10.2019 \\ accepted after revision 20.1 .2020 \\ Bibliography \\ DOI https://doi.org/10.1055/a-1132-5323 | \\ Endoscopy International Open 2020; 08: E724-E732 \\ (c) Georg Thieme Verlag KG Stuttgart · New York \\ eISSN 2196-9736 \\ Corresponding author \\ Edward Villa, John H Stroger Hospital of Cook County - \\ Gastroenterology and Hepatology, 1900 W Polk St 14th \\ Floor, Chicago Illinois 60612-3785 \\ Phone: +1-847-668-3875 \\ eespee88@gmail.com
}

Edward Villa, Matthew Stier, Kianoush Donboli, Christopher Grant Chapman, Uzma D. Siddiqui, Irving Waxman

\section{ABSTRACT}

Background and study aims Colonic lesions may not be amenable to conventional endoscopic mucosal resection (EMR) due to previous manipulation, submucosal invasion, or lesion flatness. In 2018, we described Dissection-enabled Scaffold Assisted Resection (DeSCAR) to be safe for the endoscopic resection of non-lifting or residual colonic lesions [1] In this study, we expand our original cohort to describe our expanded experience with patients undergoing DeSCAR and assess the efficacy, safety, and feasibility of DeSCAR for endoscopic resection of non-lifting or residual colonic lesions.

Patients and methods We retrospectively reviewed 57 patients from 2015-2019 who underwent DeSCAR for colonic lesions with incomplete lifting and/or previous manipulation. Cases were reviewed for location, prior manipulation, rates of successful resection, adverse events, and endoscopic follow up to assess for residual lesions.

Results Fifty-seven lesions underwent DeSCAR. Of the patients, $51 \%$ were female, and average patient age was 69 years. Lesions were located in the cecum $(n=16)$, right colon $(n=27)$, left colon $(n=10)$, and rectum $(n=4)$. Average lesion size was $27.7 \mathrm{~mm}$. Previous manipulation occurred in 54 cases ( $72 \%$ biopsy, $44 \%$ resection attempt, $18 \%$ intralesional tattoo). The technical success rate for resection of non-lifting lesions was $98 \%$. There were two delayed bleeding episodes (one required endoscopic intervention) and one small perforation (managed by endoscopic hemoclip closure). Endoscopic follow up was available in 31 patients (54\%) with no residual adenoma in 28 patients (90\% of those surveilled).

Conclusions Our expanded experience with DeSCAR demonstrates high safety, feasibility, and effectiveness for the endoscopic management of non-lifting or residual colonic lesions.

\section{Introduction}

Endoscopic resection of superficial colorectal lesions is highly effective for the removal of mucosal lesions with high rates of technical success, relatively low cost, and acceptably low adverse event rates in experienced centers [1-7]. Increased performance in the community setting has led to overall improvement in the en bloc removal of superficial colorectal lesions without the need for surgical intervention. However, for treat- ment of advanced adenomas (flat/sessile lesions greater than 2 $\mathrm{cm}$ in size) and scarred lesions, endoscopic mucosal resection (EMR) is limited given reliance on piecemeal resection techniques in the former and inability to achieve adequate lifting in the latter. This is evidenced by local recurrence rates as high as $10 \%$ to $30 \%[1,2,4-15]$.

Endoscopic submucosal dissection (ESD) overcomes some of these shortcomings while allowing for en bloc resection of large superficial gastrointestinal neoplasias. The result is im- 
proved histopathologic evaluation, assessment of deeper resection margins, and lower tumor recurrence rates with technical success rates of $88 \%$ to $96 \%$ and curative resection rates approaching $90 \%$ reported in high volume centers [8, 16-26]. ESD also has good performance characteristics and technical success rates when applied to advanced adenomas - particularly those with invasion up to the superficial muscularis propria and scarred, benign lesions not able to be removed by conventional EMR. However, because of the technical complexities of the procedure - which may result in prolonged procedure times and risk of adverse events - ESD implementation in the United States has been limited to expert, high-volume centers and has, thus, not been widely implemented in the community setting, unlike the widespread performance of EMR [4,22-26].

Hybrid resection methods - including precut EMR-that blend the principles of EMR and ESD have also proven efficacious for en bloc, resection of colorectal neoplasias and as a rescue treatment in cases of difficult ESD [27-29]. Recent comparisons of optimized hybrid versus conventional ESD have shown comparable en bloc resection rates and adverse event profiles with significantly shorter mean procedure times in the hybrid ESD groups, but, like ESD, are limited to expert centers [28, 29].

As a result of the above challenges inherent to en bloc and complete removal of these lesions, novel techniques are necessary to address these lesions, ideally ones that could be readily available, easy to adopt, and allow for complete histologic evaluation of the lesion of interest. Dissection-enabled scaffold-assisted resection (DeSCAR) is a new technique that can allow for complete resection of superficial non-lifting lesions that remain after failed lifting and/or snaring attempts because of fibrosis or submucosal invasion [1]. This hybrid, technical variation of ESD combines EMR and ESD electrosurgical principles to allow for en bloc snaring of residual, non-lifting, post-EMR islands for histopathologic review, and our group has previously demonstrated the feasibility, safety and efficacy of this technique. [1] We present our expanded experience and outcomes of the DeSCAR technique.

\section{Patients and methods}

A retrospective analysis of a prospectively collected database was used to identify patients referred for EMR of colonic lesions. From this database, cases were selected where lifting and/or snaring of the lesion was unsuccessful or incomplete and the DeSCAR technique was undertaken ( $>$ Fig. 1). All cases occurred between September 2015 and September 2019. Procedures were performed at the University of Chicago Medicine, a quaternary care, urban academic medical center. Of 367 cases where a complex colonic neoplasm was encountered, a total of 57 patients (16\%) underwent DeSCAR. A single experienced endoscopist (I.W.) performed all procedures. Lesions were selected for DeSCAR at the discretion of the endoscopist based on primary non-lifting or incomplete EMR attempts using standard submucosal lifting techniques. Location within the colon was divided into cecum, right side of the colon (including ascending and transverse colon), left side of the colon (including descending and sigmoid colon), and rectum. Exclusion criteria included the use of ESD or EMR alone and primary refer-
367 total patients underwent colonic resection for advanced colonic lesions from 2015 to 2019

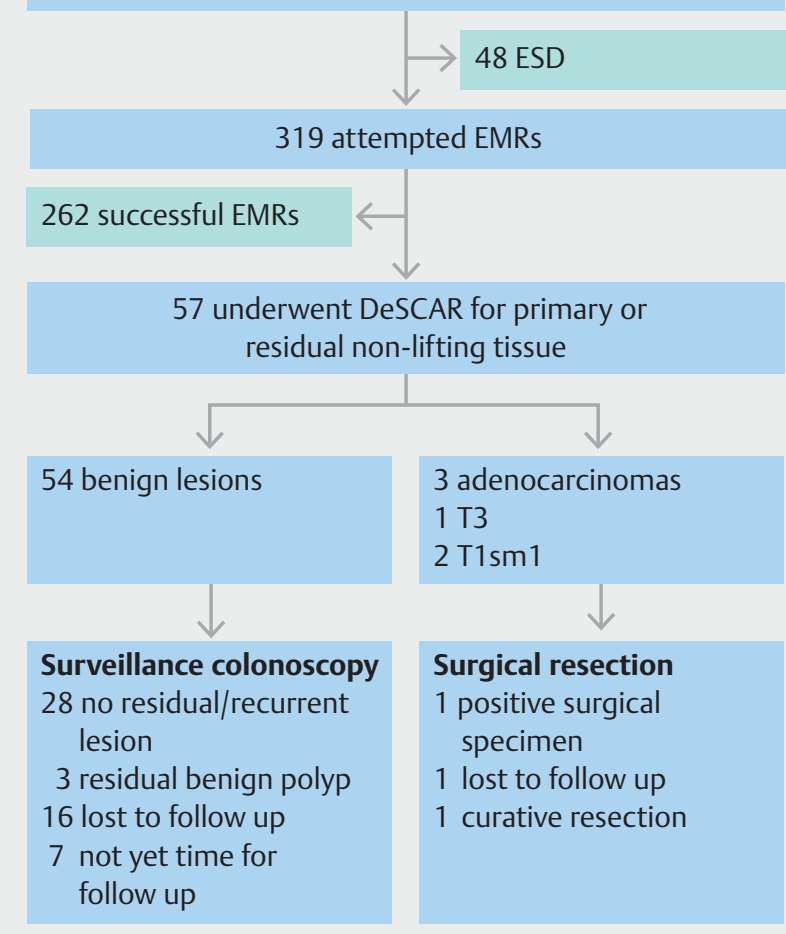

Fig. 1 Study flowchart.

ral to surgery based on invasive malignant appearance. Cases were reviewed for patient and polyp characteristics, previous manipulation, technical success rate, and adverse events. Institutional approval for the study was obtained (IRB approval number 11-0721, approved April 2012 with last renewal in June 2019).

\section{Procedural technique}

All procedures were performed on an outpatient basis, and sedation was achieved via monitored anesthesia care. Procedures used high-definition Olympus (Olympus, Tokyo, Japan) model CF190 colonoscopes or Olympus HQ190 gastroscopes. An Olympus 12 - or $15-\mathrm{mm}$ translucent cap was placed on the endoscope for better visualization of the mucosa during resection. A standard 15-mm ConMed snare (Utica, New York) was used for endoscopic mucosal resection. Either an electrosurgical DualKnife (Olympus) or the tip of the 15-mm snare was used for submucosal dissection to build the scaffold. Cautery settings were variable and case dependent. Location of the lesion was determined using anatomic landmarks and depth of endoscope insertion as appropriate. Lesion size was estimated visually using comparison with endoscopic snaring devices and/ or direct ex vivo measurement before pathology processing.

In all lesions amenable to an initial EMR attempt, a mixture of methylene blue and hetastarch solution was injected into the submucosa in order to raise the lesion. If lifting was suc- 

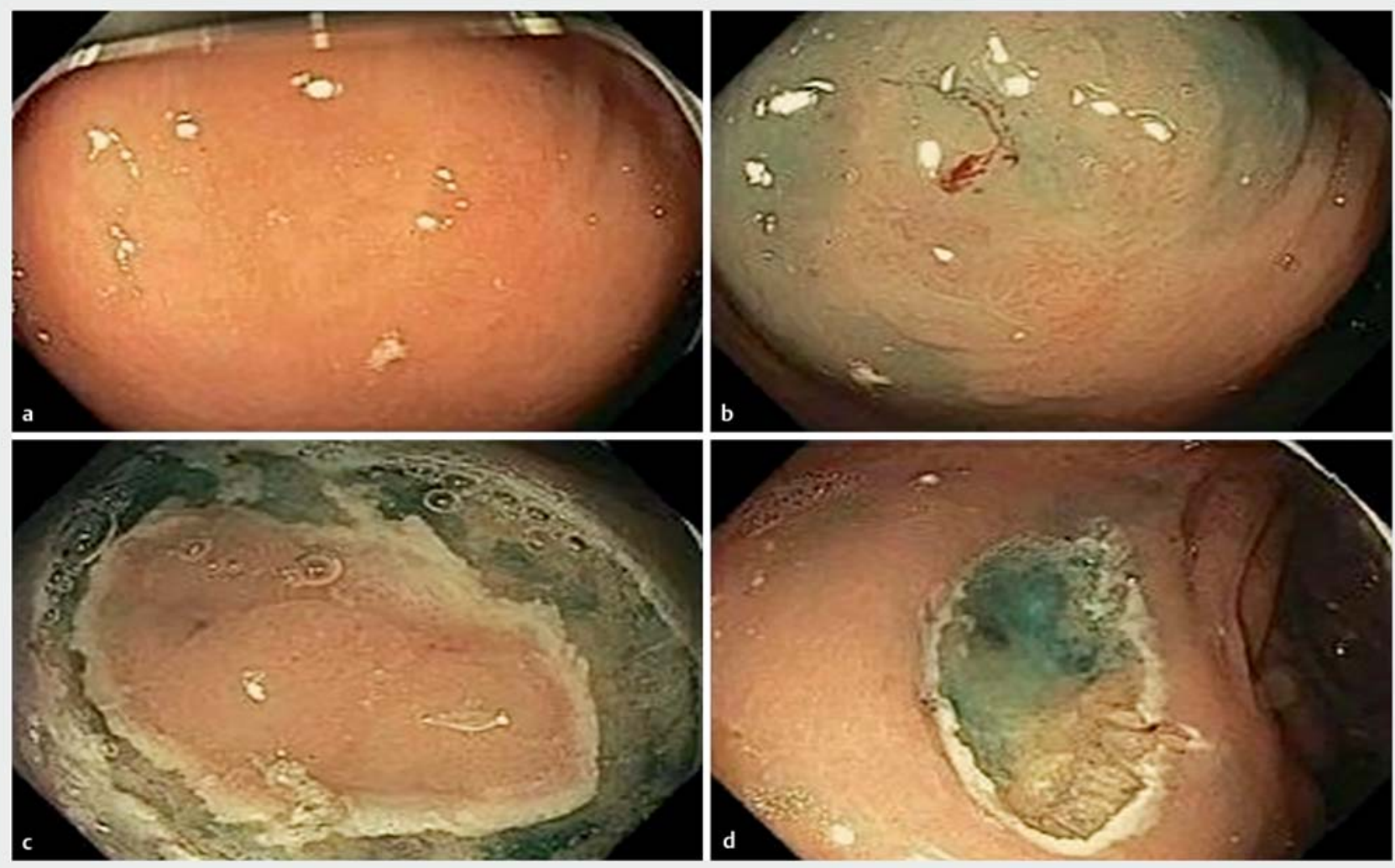

Fig. 2 Paris Classification Ila + c lesion in the right colon undergoing DeSCAR with histology shown in $>$ Fig. 3.

cessful, complete or piecemeal EMR was attempted. Any suspicious tissue remaining after multiple attempts at snare placement or displaying partial or non-lifting properties were subsequently re-injected with additional methylene blue and hetastarch. A DualKnife or the tip of a $15-\mathrm{mm}$ snare was then used for electrosurgical dissection to create a "scaffold" around the residual island of tissue, circumferentially exposing the lesion to the submucosal level. This scaffold was used to anchor the snaring device in a groove below the level of the scarred mucosa ( $\triangleright$ Fig. 2). Occasionally, a brief submucosal dissection was performed to further anchor the snare below the scarred or infiltrating portion of the lesion. The residual tissue was then removed by snare en bloc and submitted to pathology in its entirety in a separate jar. Preventative hemostasis and tissue ablation were undertaken on an as-needed basis at the discretion of the endoscopist. Technical success was defined by the endoscopist as complete resection of all endoscopically visible lesions. Adverse events directly related to the procedure were defined as immediate or delayed bleeding requiring hospitalization, transfusion or repeat procedure, and perforation requiring medical and/or procedural interventions. Follow-up endoscopy to detect recurrence was recommended and was performed by the referring physician or at our institution depending on patient preference.

\section{Outcome measurements}

The primary outcomes measured were technical success rate, presence or absence of recurrence at follow up, as well as the type and frequency of adverse events. Secondary outcomes included the diagnosis of initial EMR specimens versus diagnosis from residual tissue removed via the DeSCAR technique.

\section{Results}

In addition to our previous cohort of 29 patients who underwent DeSCAR from September 2015 through June 2017 [1], an additional 28 patients underwent DeSCAR for colonic lesions with incomplete lifting and/or snaring from July 2017 through September 2019. Cases were reviewed for location, prior manipulation, rates of successful hybrid resection, adverse events, and endoscopic follow up to assess for residual lesions. Fiftyone percent of the patients were female and $49 \%$ were male. The average age was 69 years (range $42-87$ years) ( $\bullet$ Table 1 ). Lesions were located in the cecum $(n=16,28 \%)$, right side of the colon $(n=27,47 \%)$, left side of the colon $(n=10,18 \%)$, and rectum $(n=4,7 \%)$ ( $\vee$ Table 1$)$. The mean size of the lesions was $27.7 \mathrm{~mm}$ (range $10-100 \mathrm{~mm}$ ) ( $\bullet$ Table 2 ).

Procedural indications included carcinoma $(n=2)$, high grade dysplasia $(n=4)$, tubular adenomas $(n=38)$, and serrated lesions $(n=10)$. Pathology for 3 lesions was not available because they were not sampled before DeSCAR. Previous manipulation of any kind occurred in $95 \%$ of cases. A biopsy was pre- 
- Table 1 Patient and lesion characteristics

\begin{tabular}{|c|c|}
\hline & \\
\hline Age (years) & $68.5 \pm 9.6$ \\
\hline Gender (\%F/\%M) & $51 / 49$ \\
\hline Lesion size (mm) & $27.7 \pm 16.6$ \\
\hline Size range (mm) & $10-100$ \\
\hline \multirow[t]{4}{*}{ Paris classification (n) } & $11 a-42$ \\
\hline & $11 a+c-6$ \\
\hline & Is -5 \\
\hline & $11 \mathrm{a}+\mathrm{Is}-4$ \\
\hline \multirow[t]{6}{*}{ Lesion location (n) } & Cecum/ileocecal valve - 16 \\
\hline & Right colon - 22 \\
\hline & Transverse colon - 5 \\
\hline & Left colon - 7 \\
\hline & Rectum - 4 \\
\hline & Other - 3 \\
\hline \multirow[t]{5}{*}{ Referring diagnosis (n) } & Adenocarcinoma - 2 \\
\hline & Dysplasia - 4 \\
\hline & $\begin{array}{l}\text { Tubular adenoma without advanced } \\
\text { dysplasia - } 38\end{array}$ \\
\hline & Serrated adenoma - 10 \\
\hline & Not available - 3 \\
\hline
\end{tabular}

viously performed in $72 \%$, resection was attempted in $44 \%$, and intralesion tattoo placement occurred in $18 \%$ ( $>$ Table 2). Initial EMR was attempted in $88 \%$ of cases (50 patients) before using DeSCAR for residual tissue islands. DeSCAR was used as the initial technique in $12 \%$ because of overt visual scarring and/or primary inability to lift the lesion satisfactorily for snare attempts. A DualKnife $(n=32)$ or the tip of a $15-\mathrm{mm}$ snare $(n=$ 25 ) were used to create the submucosal scaffold for all cases using the DeSCAR technique.

56 lesions in this cohort were resected endoscopically for a technical success rate of $98 \%$ with the lone unresected lesion being a T3 invasive adenocarcinoma ( $\mathbf{F i g . 3}$ ). Prior to resection of the T3 lesion - a lesion previously biopsied by the referring gastroenterologist with histopathology demonstrating tubulovillous adenoma - the 0 -lla $+\mathrm{c}$ lesion was assessed with high definition endoscopy with narrow band imaging and high magnification endoscopy lesion's pre-procedural histopathology was a tubulovillous adenoma on biopsies; the lesion was non-lifting during initial submucosal injection. On histopathologic review of the completely resected lesions there were two

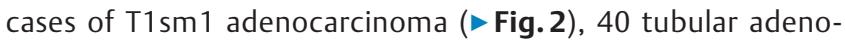
mas, 11 serrated adenomas, and three samples containing only colonic mucosa ( $\$$ Table 2 ). No patients experienced upstaging of their diagnosis based on the histology encountered in the residual island of non-lifting tissue. Three lesions demonstrated positive deep margins on histology and were referred for surgery (5\%). One of these contained a T3 rectal adenocarcinoma on surgical pathology (as mentioned above); one demonstrated no residual disease in the surgical specimen; and one patient was lost to follow-up.

At least one method of preventative hemostasis was attempted for $86 \%$ of lesions removed by DeSCAR. This included the use of a bipolar probe (47\%), metallic clips (60\%), hot biopsy forceps (5\%), and coagulation grasping forceps (4\%). When metallic clips were placed, the average number of clips deployed was 3.7. 18 patients (32\%) required two or more types of preventative hemostasis. APC was not applied in any of the cases for hemostasis or ablation of residual tissue. There were two cases of delayed bleeding (3.5\%), one of which was treated endoscopically with bipolar and hot biopsy forceps electrocautery the following day, and the other managed conservatively without a need for endoscopic intervention ( $>$ Table 2 ). There was one case of perforation (1.8\%) occurring at the time of DeSCAR, which was closed endoscopically with hemostatic clips ( $>$ Table 2 ). No other significant adverse events reported in any of the patients undergoing procedures using the DeSCAR technique. Follow-up endoscopy was recommended between 6 and 12 months and available for 31 patients (54\%). Twentyeight patients ( $90 \%$ of patients with colonoscopic surveillance) had no evidence of residual polyp, and the remaining three patients had residual non-dysplastic adenomatous polyps.

\section{Discussion}

Improved recognition of sessile and flat colorectal lesions has prompted an increase in tertiary care referrals for endoscopic resection [30-32]. However, as a result of submucosal invasion or submucosal fibrosis from previous lesion manipulation (incomplete resection attempts, extensive biopsies, or intralesional tattooing) resections even at expert centers have become more complex, given impeded lifting of those manipulated or more invasive lesions [3, 32, 33].

While generally effective as a first-line technique for the resection of colorectal neoplasia, the efficacy of EMR is best demonstrated in raised, lifting, smaller $(\leq 2 \mathrm{~cm})$ lesions in which en bloc resection is achieved [1-7]. With piecemeal approaches for larger, flat lesions, there is a decreased reliability of tissue resection with rates of residual dysplasia and local recurrence as high as $10 \%$ to $30 \%$ of cases $[1,2,4-10]$. Thus, lesion selection for EMR is important, as submucosal fibrosis - whether resulting from deep submucosal resection by high-grade benign or malignant lesions or from scarring from previous endoscopic manipulation - can limit complete, adequate resections. Lesion non-lifting during attempted submucosal injection may be a harbinger for submucosal invasion; however, reliance on this "non-lifting sign" alone has limited sensitivity for deep submucosal (sm3) invasion, especially in the setting of previous sampling or injection [2, 9,11-15]. High-risk histopathologic features - including poor differentiation, tumor depth exceeding $1000 \mu \mathrm{m}$, and lymphovascular invasion - are at higher risk of metastases to locoregional lymph nodes and are better served with surgical resection and nodal sampling [12-15]. 

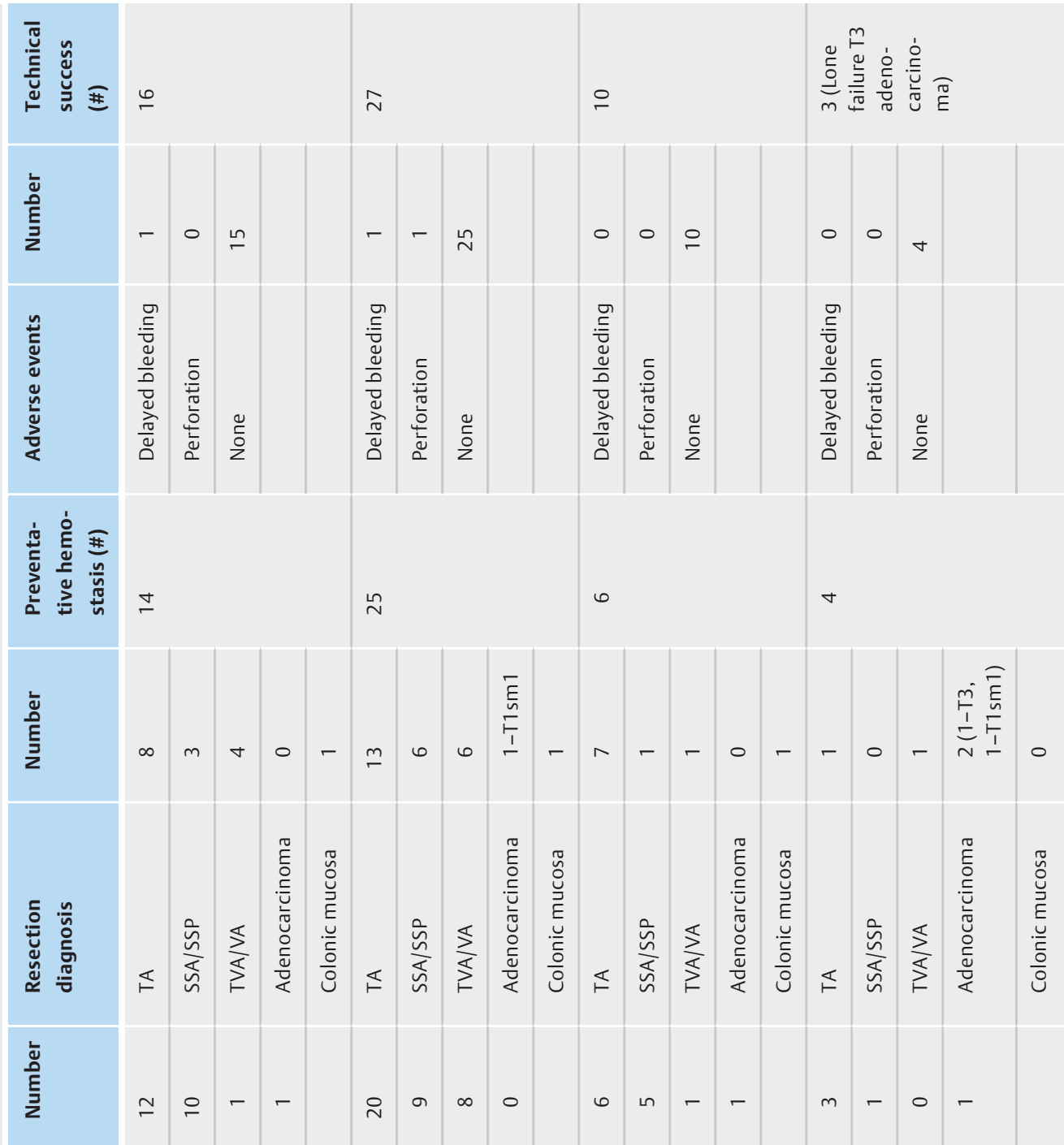

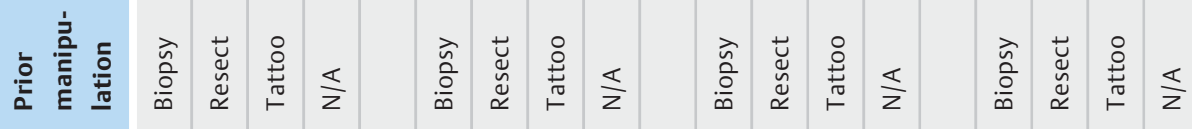

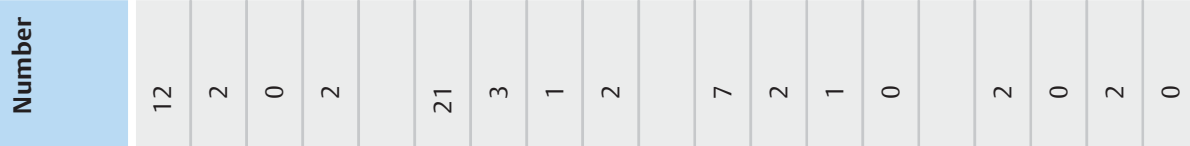

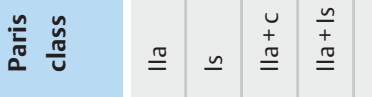

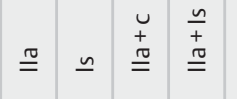

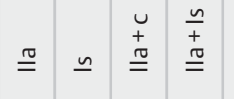

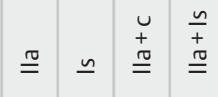

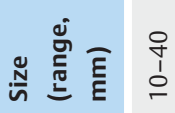

$\stackrel{8}{\frac{1}{1}}$

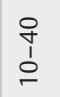

$\stackrel{\circ}{i}$

है

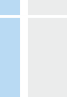

in

ฮั

స

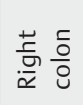

$\underbrace{\Xi}_{\mathscr{Z}}$ 

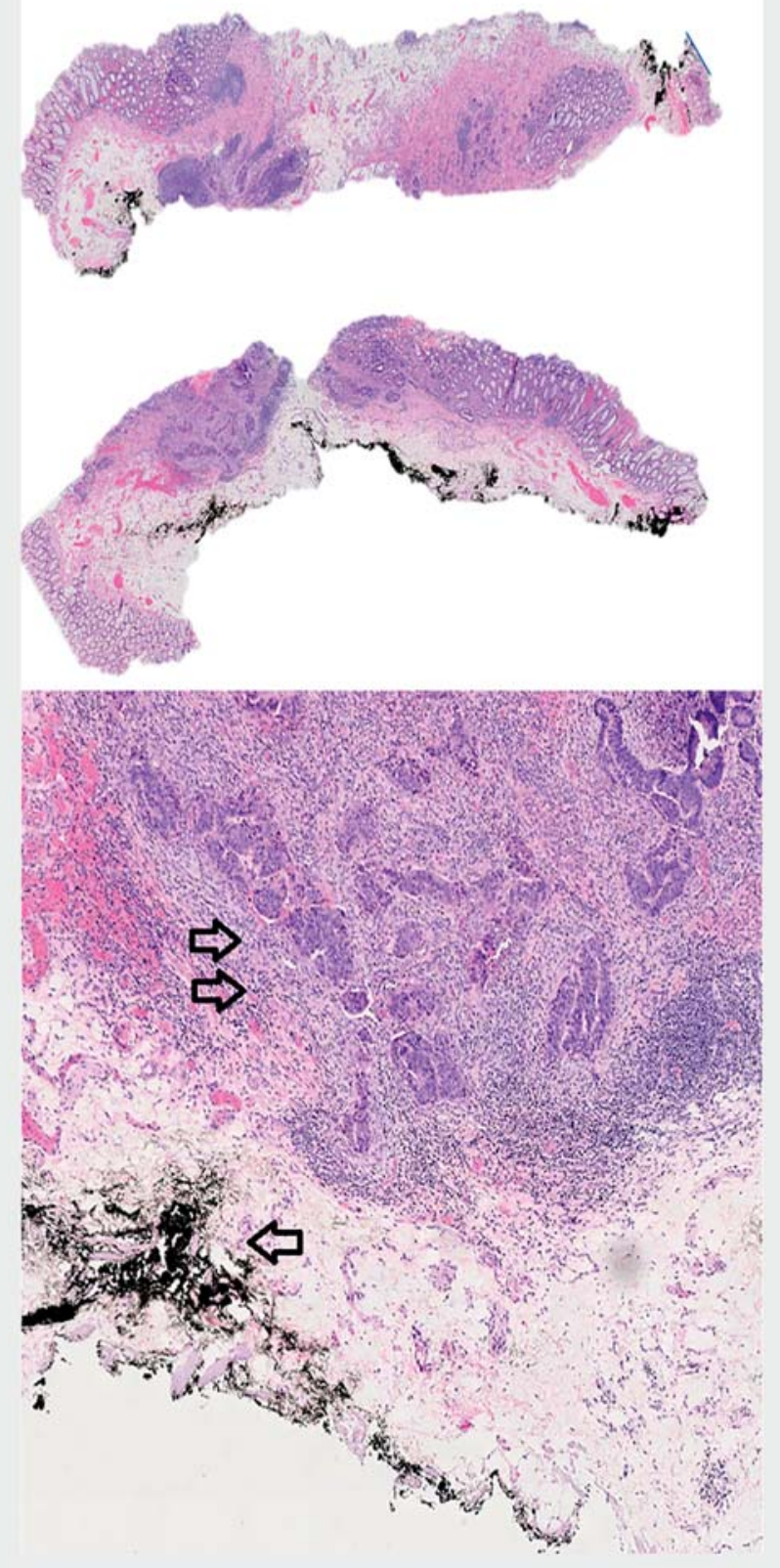

- Fig. 3 Polypectomy specimen demonstrating T1sm 1 adenocarcinoma (double arrow) after DeSCAR with intralesional tattoo (arrow) seen on histopathology.

ESD has demonstrated particular effectiveness for the resection of lesions that are otherwise not amenable to en bloc snare removal by EMR with improved histologic diagnosis, lower recurrence rates and reasonably comparable safety outcomes for the management of tumors with submucosal involvement. This approach has continued to diffuse into major American and European institutions with expert center complete resection rates as high as $88 \%$ to $96 \%$ and curative resection near $90 \%$, outperforming the same performance outcomes of EMR [2, 8,16-24].
While other prospective and European studies have been less optimistic, when applied to well-selected lesions, en bloc and curative resections remain high and compare favorably to EMR and surgical interventions [16,17,22,23,25]. Although the adoption of advanced endoscopic dissection techniques represents an important advancement in the field of therapeutic endoscopy, in Western centers these procedures are limited to specialized, high-volume centers due to the technical complexities, prolonged procedure times, and risk of adverse events [2, 4, 21-24, 26].

Hybrid endoscopic resection techniques that combine the ease and safety profile of EMR with the complete resection potential of ESD are also effective strategies for en bloc resection of colorectal lesions and offer a reasonable rescue treatment for cases of difficult ESD [27]. A more recent prospective, randomized controlled study comparing optimized hybrid ESD to standard ESD demonstrated similar en bloc resection rates among both groups ( $94.1 \%$ vs $100 \% ; P=.493$ ) with shorter procedure duration in the hybrid ESD group while maintaining a comparable adverse event rate profile [28, 29].

Endoscopic full-thickness resection (EFTR) is a newer technique wherein an over-the-scope clip is deployed over an adequately grasped/suctioned lesion (typically through a transparent cap), and the lesion is subsequently removed with a snare [34-39]. The technical success of EFTR for benign, recurrent adenomas with non-lifting sign, advanced histopathological findings, or submucosal involvement approached $75 \%$ to $100 \%$ and histologically complete resection (R0) rates of $80 \%$ to $93 \%$ has led to curative resection of advanced benign and malignant lesions, obviating the need for surgery, particularly for lesions limited to the superficial submucosa without evidence of lymphovascular invasion or poor grade of differentiation [35-39]. Applied to previously manipulated lesions, R0 resection rates approach $89 \%$ [35-39]. However, while surgery can be avoided in many cases in which EFTR is used, R0 resection rates are lower for lesions greater than $2 \mathrm{~cm}$ in size $(33 \%-$ $87 \%$ ) and those that contain adenocarcinoma (67\%), and a total complication rate of $5 \%-15 \%$ was observed in studies published to date [34-39]. A hybrid EMR-EFTR technique - wherein the lifting portions of the lesion are removed by EMR and the nonlifting portions removed by EFTR - was developed to address the size limitation of EFTR and was applied to colonic lesions with a median size of $35.5 \mathrm{~mm}$ with $100 \% \mathrm{R} 0$ resection rate, no residual or recurrent adenoma at 3-month follow up, and no reported complications [40]. However, this was a limited cohort of 10 patients, and this approach warrants further study.

Argon plasma coagulation (APC) is a less invasive endoscopic method for managing residual neoplasia after unsuccessful snare removal during EMR and is attractive because of its safety profile, ease of use, and ubiquitous availability at most endoscopy centers [41]. However, data are mixed on the efficacy as a primary therapy for colorectal neoplasia, as multiple studies have suggested that APC is an independent risk factor for recurrent neoplasia, occurring in as high as $59 \%$ of cases $[6,10$, 42].

Cold or hot avulsion techniques - that is, grasping of nonlifting tissue using forceps with or without the application 
high-frequency cutting current and mechanical traction - have been described as methods to remove difficult to resect neoplastic tissue and has demonstrated more favorable rates of local recurrence when compared to APC in some studies [42-46]. This technique is particularly useful for residual lesions unable to be removed with conventional EMR whether due to difficult locations or inability to capture the lesion with the snare. Incomplete histologic evaluation of the non-lifting central scardue to tissue fragmentation and sample degradation due to cautery effects, the latter seen in the case of hot avulsion - as well as a non-negligible tumor recurrence rate remaining over $10 \%$ are the main limitations of these techniques [46].

While the above techniques used to endoscopically remove scarred, non-lifting colorectal lesions are feasible strategies for resection of these historically difficult-to-resect colorectal lesions, the improved recognition of sessile and flat colorectal lesions; limitations of EMR for large, sessile colorectal lesions; complexities associated with standard ESD and EMR-ESD techniques; lack of widespread adoption of ESD or EFTR; and lack of consistent efficacy of ablative or avulsion therapies have unsurprisingly led to an increase in tertiary care referrals for endoscopic resection of colorectal lesions [3, 31, 32]. However, as a result of submucosal invasion or submucosal fibrosis from previous lesion manipulation (incomplete resection attempts, extensive biopsies, or intralesional tattooing) resections even at expert centers have become more complex, given impeded lifting of those manipulated or more invasive lesions [3, 33,4143].

DeSCAR is a hybrid endoscopic resection technique blending the ease and speed of EMR with a minimal submucosal dissection, allowing for en bloc, intact pathologic specimens. This results in improved diagnostic accuracy for the identification of advanced disease while assessing the adequacy of resection. This improvement in removal of non-lifting lesions also has the potential to increase rates of curative resection over piecemeal EMR. Similar to other techniques utilizing pre-cut mucosal and submucosal tracts - techniques utilizing biopsy forceps, grasp or snare devices through the second channel of a dual channel endoscope, or tissue anchoring/traction-assisted methods the achieved rates of complete resection were slightly above conventional EMR with comparable safety and limited additional procedural time [47-52]. However, these techniques have not resulted in equivalent complete resection rates as ESD [50-52]. Also, none of these studies were applied in patients with residual invasive and/or fibrosed areas of tissue after primary piecemeal EMR attempts.

In our study, the technical success rate for resection of residual or primary non-lifting tissue using DeSCAR was $98 \%$, demonstrating its feasibility in expert hands and continued technical success in our additional patient cohort. It is important to note that our observed complete resection rates are noticeably higher than those reported in EMR among non-lifting, scarred lesions, which have been historically difficult lesions to resect endoscopically and were often referred for surgery in the past. This observation is likely a result of the margination of the lesion with a scaffold that extends to the level of the submucosa, allowing the snare to capture the entire lesion at a deeper level than what is capable with conventional EMR. As a result, the recurrent adenoma rate compares superiorly to piecemeal EMR and EMR for non-lifting, scarred or deeply penetrating lesions and is comparable to published data for both ESD and EFTR. Our data are limited by our small overall patient cohort of 57 patients and our limited 12-month follow up of 31 patients (54\% of our total cohort). Anecdotally, our procedure times were shorter than conventional ESD for similarly sized lesions, but we did not directly record our DeSCAR procedure lengths. Further experience and follow up are needed in order to comment further on the efficacy of DeSCAR, but our results with an expanded cohort continues to support the feasibility and efficacy of this technique for resection of non-lifting lesions.

There were only three complications observed (5\%), including two delayed bleeding events - one of which was promptly treated with standard endoscopic hemostatic therapies; the other was managed conservatively without the need for endoscopic therapy - and one case of perforation at the time of DeSCAR that was treated promptly with endoscopic hemoclip closure. This demonstrates a promising safety profile with delayed bleeding and perforation rates comparable with current EMR techniques. Three patients who continued in surveillance at our institution ( $10 \%$ of patients undergoing surveillance) had residual, non-dysplastic adenomatous tissue, which is comparable to previously published ESD data.

\section{Conclusion}

For removal of large sessile gastrointestinal lesions, piecemeal EMR continues to be a commonly practiced technique but is limited in the setting of previously manipulated or deeply invasive neoplasias. Without universal availability or expertise, ESD is also limited in the Western hemisphere and may not be technically feasible in many cases. While application of DeSCAR in this study was limited to a small number of cases that were evaluated in a retrospective fashion, our expanded experience with DeSCAR continues to demonstrate a strong safety profile and feasibility for en bloc removal of non-lifting colorectal neoplasias and offers the possibility for more complete eradication of neoplastic tissue with improved histopathologic evaluation. Further high-quality research is needed to determine if this method can offer decreased recurrence rates and higher diagnostic accuracy as compared to other ablative modalities as well. Improved awareness within the GI community regarding avoidance of partial mucosal resection and appropriate tattoo location before specialist referral remains necessary.

\section{Competing interests}

Dr. Waxman is affiliated with Boston Scientific, Cook Medical, Medtronic, and Olympus. Dr. Siddiqui is affiliated with Boston Scientific, ConMed, Medtronic, and Olympus. Dr. Chapman is affiliated with Apollo Endosurgery, Boston Scientific, and Olympus 


\section{References}

[1] Stier M, Chapman C, Kreitman A et al. Dissection-enabled scaffoldassisted resection (DeSCAR): A novel technique for resection of residual or non-lifting $\mathrm{GI}$ neoplasia of the colon (with video). Gastrointest Endosc 2018; 87: 843-851

[2] Ferlitsch M, Moss A, Hassan C et al. Colorectal polypectomy and endoscopic mucosal resection (EMR): European Society of Gastrointestinal Endoscopy (ESGE) clinical guideline. Endoscopy 2017; 49: 270-297

[3] Buchner AM, Guarner-Argente C, Ginsberg GG. Outcomes of EMR of defiant colorectal lesions directed to an endoscopy referral center. Gastrointest Endosc 2012; 76: 255-263

[4] Burgess NG, Bourke MJ. Endoscopic resection of colorectal lesions: the narrowing divide between East and West. Dig Endosc 2016; 28 : 296-305

[5] Hassan C, Repici A, Sharma P et al. Efficacy and safety of endoscopic resection of large colorectal polyps: a systematic review and metaanalysis. Gut 2016; 65: 806-820

[6] Moss A, Williams S], Hourigan LF et al. Long-term adenoma recurrence following wide-field endoscopic mucosal resection (WF-EMR) for advanced colonic mucosal neoplasia is infrequent: results and risk factors in 1000 cases from the Australian Colonic EMR (ACE) study. Gut 2015; 64: 57-65

[7] Pellise M, Burgess NG, Tutticci N et al. Endoscopic mucosal resection for large serrated lesions in comparison with adenomas: a prospective multicentre study of 2000 lesions. Gut 2017; 66: 644-653

[8] Klein A, Bourke M]. Advanced polypectomy and resection techniques. Gastrointest Endosc Clin N Am 2015; 25: 303-333

[9] Moss A, Bourke M], Williams S] et al. Endoscopic mucosal resection outcomes and prediction of submucosal cancer from advanced colonic mucosal neoplasia. Gastroenterology 2011; 140: 1909-1918

[10] Rex KD, Vemulapalli KC, Rex DK. Recurrence rates after EMR of large sessile serrated polyps. Gastrointest Endosc 2015; 82: 538-541

[11] Han KS, Sohn DK, Choi DH et al. Prolongation of the period between biopsy and EMR can influence the nonlifting sign in endoscopically resectable colorectal cancers. Gastrointest Endosc 2008; 67: 97-102

[12] Uno Y, Munakata A. The non-lifting sign of invasive colon cancer. Gastrointest Endosc 1994; 40: 485-489

[13] Matsuda T, Parra-Blanco A, Saito Y et al. Assessment of likelihood of submucosal invasion in non-polypoid colorectal neoplasms. Gastrointest Endosc Clin N Am 2010; 20: 487-96

[14] Nakadoi K, Tanaka S, Kanao H et al. Management of T1 colorectal carcinoma with special reference to criteria for curative endoscopic resection. J Gastroenterol Hepatol 2012; 27: 1057-1062

[15] Nascimbeni R, Burgart LJ, Nivatvongs $S$ et al. Risk of lymph node metastasis in T1 carcinoma of the colon and rectum. Dis Colon Rectum 2002; 45: 200-206

[16] Ikematsu $H$, Yoda Y, Matsuda T et al. Long-term outcomes after resection for submucosal invasive colorectal cancers. Gastroenterology 2013; 144: 551-559; quiz e514

[17] Kiriyama S, Saito Y, Yamamoto S et al. Comparison of endoscopic submucosal dissection with laparoscopic-assisted colorectal surgery for early-stage colorectal cancer: a retrospective analysis. Endoscopy 2012; 44: 1024-1030

[18] Kitajima K, Fujimori T, Fujii S et al. Correlations between lymph node metastasis and depth of submucosal invasion in submucosal invasive colorectal carcinoma: a Japanese collaborative study. J Gastroenterol 2004; 39: 534-543

[19] Repici A, Hassan C, De Paula Pessoa D et al. Efficacy and safety of endoscopic submucosal dissection for colorectal neoplasia: a systematic review. Endoscopy 2012; 44: 137-150
[20] Saito Y, Matsuda T, Fujii T. Endoscopic submucosal dissection of nonpolypoid colorectal neoplasms. Gastrointest Endosc Clin N Am 2010; 20: $515-524$

[21] Tanaka S, Oka S, Kaneko I et al. Endoscopic submucosal dissection for colorectal neoplasia: possibility of standardization. Gastrointest Endosc 2007; 66: 100-107

[22] Fujiya M, Tanaka K, Dokoshi T et al. Efficacy and adverse events of EMR and endoscopic submucosal dissection for the treatment of coIon neoplasms: a meta-analysis of studies comparing EMR and endoscopic submucosal dissection. Gastrointest Endosc 2015; 81: 583595

[23] Saito Y, Fukuzawa M, Matsuda T et al. Clinical outcome of endoscopic submucosal dissection versus endoscopic mucosal resection of large colorectal tumors as determined by curative resection. Surg Endosc 2010; 24: 343-352

[24] Saito Y, Uraoka T, Yamaguchi Y et al. A prospective, multicenter study of 1111 colorectal endoscopic submucosal dissections (with video). Gastrointest Endosc 2010; 72: 1217-1225

[25] Currie AC, Askari A, Rao C et al. The potential impact of local excision for $\mathrm{T} 1$ colonic cancer in elderly and comorbid populations: a decision analysis. Gastrointest Endosc 2016; 84: 986-994

[26] Backes Y, Moons LM, van Bergeijk JD et al. Endoscopic mucosal resection (EMR) versus endoscopic submucosal dissection (ESD) for resection of large distal non-pedunculated colorectal adenomas (MATILDA-trial): rationale and design of a multicenter randomized clinical trial. BMC Gastroenterol 2016; 16: 56

[27] Okamoto K, Muguruma N, Kagemoto K et al. Efficacy of hybrid endoscopic submucosal dissection (ESD) as a rescue treatment in difficult colorectal ESD cases. Dig Endosc 2017; 29: 45-52

[28] Bae JH, Yang DH, Lee $S$ et al. Optimized hybrid endoscopic submucosal dissection for colorectal tumors: a randomized controlled trial. Gastrointest Endosc 2016; 83: 584-592

[29] Gorospe EC, Wong Kee Song LM. Hybrid endoscopic submucosal dissection in the colon: Cutting corners or trimming fat? Gastrointest Endosc 2016; 83: 593-595

[30] Friedland S, Banerjee S, Kochar R et al. Outcomes of repeat colonoscopy in patients with polyps referred for surgery without biopsy proven cancer. Gastrointest Endosc 2014; 79: 101-107

[31] Raju GS, Lum PJ, Ross WA et al. Outcome of EMR as an alternative to surgery in patients with complex colon polyps. Gastrointest Endosc 2016; 84: 315-325

[32] Friedland S, Shelton A, Kothari S et al. Endoscopic management of nonlifting colon polyps. Diagn Ther Endosc 2013; 2013: 412936

[33] Kim HG, Thosani N, Banerjee $S$ et al. Effect of prior biopsy sampling, tattoo placement, and snare sampling on endoscopic resection of large nonpedunculated colorectal lesions. Gastrointest Endosc 2015; 81: $204-213$

[34] Shafqet M, Caruso C, Diehl D. Treatment of neoplastic colonic lesions using the full-thickness resection device. VideoGIE 2019; 4: 535-538

[35] Albrecht H, Raithel M, Braun A et al. Endoscopic full-thickness resection (EFTR) in the lower gastrointestinal tract. Tech Coloprectol 2019; 23: 957-963

[36] Velegraki M, Trikola A, Vasiliadis K et al. Endoscopic full-thickness resection of colorectal lesions with the full-thickness resection device: Clinical experience from two referral centers in Greece. Ann Gastroenterol 2019; 32: 482-488

[37] Aepli P, Criblez D, Baumeler S et al. Endoscopic full thickness resection (EFTR) of colorectal neoplasms with the Full Thickness Resection Device (FTRD): Clinical experience from two tertiary referral centers in Switzerland. United European Gastroenterol J 2018; 6: 463-470

[38] Rajan E, Wong Kee Song L. Endoscopic full thickness resection. Gastroenterol 2018; 154: 1925-1937 
[39] Richter-Schrag H, Walker C, Thimme R et al. Full thickness resection device (FTRD). Experience and outcome for benign neoplasms of the rectum and colon. Chirurg 2016; 87: 316-325

[40] Meier B, Caca K, Schmidt A. Hybrid endoscopic mucosal resection and full-thickness resection: A new approach for resection of large nonlifting colorectal adenomas (with video). Surg Endosc 2017; 31: 4268-4274

[41] Tsiamoulos ZP, Bourikas LA, Saunders BP. Endoscopic mucosal ablation: a new argon plasma coagulation/injection technique to assist complete resection of recurrent, fibrotic colon polyps (with video). Gastrointest Endosc 2012; 75: 400-404

[42] Holmes I, Kim HG, Yang DH et al. Avulsion is superior to argon plasma coagulation for treatment of visible residual neoplasia during EMR of colorectal polyps (with videos). Gastrointest Endosc 2016; 84: 822829

[43] Watanabe T, Muro K, Ajioka Y et al. Japanese Society for Cancer of the Colon and Rectum (JSCCR) guidelines 2016 for the treatment of colorectal cancer. Int J Clin Oncol Epub 2017: doi:10.1007/s10147017-1101-6

[44] Andrawes S, Haber G. Avulsion: a novel technique to achieve complete resection of difficult colon polyps. Gastrointest Endosc 2014; 80: $167-168$

[45] Veerappan SG, Ormonde D, Yusoff IF et al. Hot avulsion: a modification of an existing technique for management of nonlifting areas of a polyp (with video). Gastrointest Endosc 2014; 80: 884-888
[46] Haber GB. Residual visible neoplasia: approaches to overt and occult polyp fragments. Gastrointest Endosc 2016; 84: 830-832

[47] Miyamoto S, Muto M, Hamamoto Y et al. A new technique for endoscopic mucosal resection with an insulated-tip electrosurgical knife improves the completeness of resection of intramucosal gastric neoplasms. Gastrointest Endosc 2002; 55: 576-581

[48] Moss A, Bourke M], Tran K et al. Lesion isolation by circumferential submucosal incision prior to endoscopic mucosal resection (CSI-EMR) substantially improves en bloc resection rates for $40-\mathrm{mm}$ colonic lesions. Endoscopy 2010; 42: 400-404

[49] Sakamoto T, Matsuda T, Nakajima T et al. Efficacy of endoscopic mucosal resection with circumferential incision for patients with large colorectal tumors. Clin Gastroenterol Hepatol 2012; 10: 22-26

[50] Sold M, Kähler G. Improved techniques for endoscopic mucosal resection (EMR) in colorectal adenoma. Viszeralmedizin 2014; 30: $33-$ 38

[51] Dauser B, Winkler T, Salehi B et al. Traction-assisted endoscopic mucosal resection for polypectomy in the large intestine. World J Gastroenterol 2010; 16: 5462-5466

[52] Lee E], Lee JB, Lee SH et al. Endoscopic treatment of large colorectal tumors: comparison of endoscopic mucosal resection, endoscopic mucosal resection-precutting, and endoscopic submucosal dissection. Surg Endosc 2012; 26: 2220-2230 